\title{
Kinematic Errors Identification and Compensation Method Analysis of the Machining Robots
}

\author{
Ya Zhang \\ Zhejiang University of Science and technology, Hangzhou, 310027, China \\ yazhang1982@126.com
}

\begin{abstract}
Keywords: Kinematic Errors; Machining Robots; Identification; Compensation
\end{abstract}
\begin{abstract}
Industrial robots were widely used in the area of stacking, handling, welding because of their good repeatability position accuracy. When the industrial robot is applied in the manufacturing area, they can do cutting work instead of the machine tool. However, the error of the machining robot is comparatively large in cutting process, the error measurement and identification process is complicated, and the error compensation is difficult. The identification and compensation method of the machining errors of machining robot are analyzed in this paper. This analysis can support a foundation and reference for improving the kinematic errors identification and compensation method of the machining robot.
\end{abstract}

\section{Introduction}

Now, industrial robots were widely used in the simple tasks in the industry, such as stacking, handling, welding, and so on. In these areas, the task trajectory is relatively simple, and the robot can be programmed with a "teaching" model. In the "teaching" model, the repeatability position accuracy of the industrial robots is high enough to satisfy the requirement of these tasks. By the development of the industrial robots, they are applied in the manufacturing area more and more. The industrial robots even can do the cutting work instead of CNC machine tool in the machining area. In the fields of aerospace, shipbuilding, high-speed rail train, etc., many cutting works, such as making holes, grinding and milling, are required to accomplish at the assembly site. The parts which need to be manufactured are usually very large. Traditional multi-axis NC machining center, with a large floor area and low flexibility, is often unable to adapt to the on-site machining requirements of such large-scale complex parts. In these occasions, the machining robots have more advantages to do these cutting task instead of CNC machine tools. The operating range of the robot is larger than CNC machine tools, and the installation space of the robot is smaller. The economic benefits and flexibility of the robots are also better than CNC machine tools. However, the machining precision of the robot is much lower than CNC machine tool because of the factor of low stiffness. It needs to improve the absolute positioning precision by some technical means for the application of machining robots. One of the methods to improve the machining accuracy usually be adopted is the kinematic errors identification and compensation method.

\section{Input Trajectory Method in the Off-Line Programming of the Machining Robots}

In the task of complex parts cutting, the task trajectory is relative complex, and the traditional "teaching" model of the robot cannot meet the requirement of programming. The robot must be able to work in the "off-line programming" model. The development of offline programming technology has promoted the application of industrial robots in the cutting task. Off-line programming is a programming method which needs to establish a geometric model of the robot and its environment by using computer graphics firstly. Then, some planning algorithms were applied to control and operate the model to complete a trajectory planning in the off-line situation. Relative to the "teaching" programming, off-line programming has a higher requirement on the absolute positioning accuracy of the robot. The absolute positioning error should be identified and compensated in the off-line programming of the machining robots. In the mode of "off-line" programming, if the kinematic errors of the machining robots were 
identified from the mathematical model which was already established according to the structure of the robots, then the "input trajectory method" can be used in the compensation of the absolute positioning error.

\section{The Method of Improving Machining Accuracy by Kinematic Errors Identification and Compensation}

In order to use the input trajectory method to compensate the absolute positioning errors in the off-line programming of the machining robots, the kinematic errors of the robots should be identified. Many scholars have proposed some error modeling and compensation methods for the problem of the low absolute positioning accuracy robots. Zheng Kunming et al. [1] established a soft body system error model based on the spatial finite element theory according to the elastic deformation characteristics of flexible members. Hou Xiaoyu et al. [2] established the rigid-flexible coupled dynamics model of the robot according to the deformation of the connecting rod and joint. De Backer et al. [3] proposed a dynamic deformation model, and predicted the tool offset in real time and compensated the errors online. Guillo et al. [4] calculated the positional deviation of the mixing head according to the external load measured by the internal pressure sensor. Where, the part of the position deviation value was used as the compensation amount to the robot to perform real-time position compensation. Schneider et al. [5] used an optical measurement system to track robots and micro-actuators online, and combined traditional industrial robots with external piezoelectric drive compensation mechanisms to achieve online correction of relative position errors between tools and workpieces. Liu Shuanglong et al. [6] proposed a robot positioning accuracy compensation method based on mechanical joint feedback. By installing an absolute grating scale at the joint of the robot and introducing the joint servo into the robot's control, the closed-loop control of the robot joint can be realized to reduce the influence of joint error on the end position and improve the positioning accuracy of the joint.

\section{Discussion}

The above research methods usually use some precise external measuring equipment to measure the errors of the machining robots. The installation operation of these instruments is relatively complicated and the technical requirements to the people are high, which makes the process of the error identification and compensation of the machining robots be difficult and costly, which is not conducive to popularization and application.

According to these difficulties in the research now, new methods to study the kinematic errors identification and compensation should be developed. In recent years, the Machining tests method has been widely used for multi-axis machine tool error identification [7-10]. The identification of the errors by the workpiece cutting should be an efficient error identification method for machining robot in theory. If the mathematical relationship of the parameters error of the machining robots and the machining errors of the workpiece according to the mathematical model of the robots, then the parameters error of the machining robots can be identified from the machining errors of the workpiece by certain error separation method.

\section{Conclusion}

The main reason of the lower machining accuracy is that the stiffness of the robot is weaker than the CNC machine tool. The error of the machining robot is comparatively large in offline programming, the error measurement and identification process is complicated, and the error compensation is difficult. According to the topology structure of the robot, establish the stiffness model, which contains the geometric parameter errors. The key technology of the machining robot is to improve machining accuracy from the model. The methods of improving the kinematic errors of the machining robot by identifying and compensating the kinematic errors of the industrial robot were analyzed in this paper. 
This analysis can support a foundation and reference for improving the accuracy of the machining robot.

\section{Acknowledgment}

This work was financially supported by Startup Foundation of Zhejiang University of Science and Technology (F701102H02).

\section{References}

[1] ZHENG Kun-ming, ZHANG Qiu-ju. Error modeling and compensation analysis of Delta robot rigid-flexible hybrid position [J]. Journal of machine design, 2017, 34(1):52-61.

[2] HOU Xiao-yu, ZHU Hua-bing, JIANG Lei Analysis of Pose Error of 6 R Serial R obot on R igid-flexible Coupling [J]. Modular Machine Tool \& Automatic Manufacturing Technique, 2018, 1 : 51-55.

[3]DE BACKER J, BOLMSJÖ G. Deflection model for robotic friction stir welding[J]. Industrial Robot: An International Journal, 2014, 41(4): 365-372.

[4] GUILLO M, DUBOURG L. Impact \& improvement of tool deviation in friction stir welding: Weld quality \& real-time compensation on an industrial robot[J]. Robotics and Computer-Integrated Manufacturing, 2016, 39: 22-31.

[5] SCHNEIDER U, MOMENI-K M, ANSALONI M, et al. Stiffness modeling of industrial robots for deformation compensation in machining[C]// Proceedings of IEEE/RSJ International Conference on Intelligent Robots and Systems. IEEE, 2014: 4464-4469.

[6] LIU Shuanglong TIAN Wei HE Xiaoxu TAN Hong LIAO Wenhe ZHANG Lin. Accuracy Compensation Technology for Robot Based on Mechanical Joint Feedback [J], Aeronautical Manufacturing Technology, 2018, 61(4): 60-64, 72

[7]Yoshitaka Morimoto, Keisuke Nakato, and Motoshi Gontani. Accuracy Evaluation of 5-Axis Machining Center Based on Measurements of Machined Workpiece-Evaluation of Accuracy of 5-Axis Controlled Machining Center[J]. Int. J. of Automation Technology, 2012. 6(5): 675-681.

[8]Zhang Ya, Fu Jianzhong, Chen Zichen. Machining tests to identify kinematic errors of machine tool table rotation axis based on sensitive directions[J]. International journal of advanced manufacturing technology, 2013, 67(1):495-500.

[9]Zhang Ya, Fu Jianzhong, Chen Zichen. Identification of Kinematic Errors of Five-axis Machine Tool Trunnion Axis from Finished Test Piece[J], Chinese Journal of Mechanical Engineering, 2014, 27(5):999-1007.

[10]Z Jiang, X Tang, X Zhou, S Zheng. Machining tests for identification of location errors on five-axis machine tools with a tilting head[J], International Journal of Advanced Manufacturing Technology, 2015, 79(1):245-254. 